\title{
Second Refinement of Jacobi Iterative Method for Solving Linear System of Equations
}

\author{
Tesfaye Kebede Eneyew, Gurju Awgichew, Eshetu Haile and Gashaye Dessalew Abie
}

\begin{abstract}
In this paper, the new method called secondrefinement of Jacobi (SRJ) method for solving linear system of equations is proposed. The method can be used to solve ODE and PDE problems where the problems are reduced to linear system of equations with coefficient matrices which are strictly diagonally dominant (SDD) or symmetric positive definite matrices (SPD) or M-matrices. In this case, our new method minimizes the number of iterations as well as spectral radius and increases rate of convergence. Few numerical examples are considered to show the efficiency of SRJ over Jacobi (J) and refinement of Jacobi $(\mathrm{RJ})$ methods.

Index Terms-Jacobi Iterative method (J), Refinement of Jacobi method (RJ), Second-Refinement of Jacobi (SRJ), Symmetric positive definite Matrix (SPD), Strictly Diagonally Dominant Matrix (SDD).
\end{abstract}

\section{INTRODUCTION}

$\mathbf{S}$ YSTEMS of linear equations arise in a large number of areas both directly in modeling physical situations and indirectly in the numerical solutions of the other mathematical models. These applications occur in all areas of the physical, biological, social science and engineering etc [1]. The solution of system of equations can be obtained either by direct method or iterative method. One of the earliest iterative methods is Jacobi iterative method named after German mathematician Carl Gustav Jacob Jacobi (1804 - 1851). It was originally considered by Jacobi (1845), but Geiringer (1949) calls it the method of simultaneous displacement, and Keller (1958) calls it the Richardson iterative method. It should be noted that total-step method is a translation of the German word "Gesamtschrittverfahrem" [2].

Recently, Jacobi iterative method can be modified in three ways as Generalized Jacobi, Refinement of Jacobi and Extrapolated (Acceleration) of Jacobi (JOR) method. The Generalized Jacobi method developed by Davod Khojasteh Salkuyeh (2007) and tried to check the convergence by considering SDD and M-matrices. The RJ method developed by Farhad Naeimi Dafchahi (2008) and the convergence is checked by considering SDD and 2-Cyclic matrices. The GJ method was also modified as Refinement of GJ (RGJ) by V. B. Kumar Vatti and Genanew Gofe Gonfa (2011) and checked the convergence by considering SDD, M-matrix and 2-Cyclic matrices. Generalized Jacobi method is a few modification of Jacobi

Manuscript received September 8, 2018; accepted April 27, 2019.

The authors are with the Department of Mathematics, Bahir Dar University, Bahir Dar, Ethiopia. E-mails: tk_ke@yahoo.com, fevenjerry@gmail.com, eshetuhg@gmail.com, gashayedessalew21@gmail. com

G.D. Abie is also with the Department of Mathematics, University of Gondar, Gondar, Ethiopia. iterative method and refinement of generalized Jacobi method is similarly a few modification of generalized Jacobi iterative method. They all tried to minimize the number of iterations and spectral radius and increases rate of convergence. But still not enough, so we need to modify the refinement of Jacobi iterative method as second refinement of Jacobi iterative (SRJ) method to minimize the number of iterations and spectral radius.

Large and sparse linear systems of the form

$$
A x=b
$$

where $A=\left(a_{i j}\right)$ is a nonsingular real matrix of order $n, b$ is a given $n$-dimensional real vector and $x$ is an $n$-dimensional vector to be determined, can be computed by iterative methods based on splitting $A$ into $A=D-L-U$, where $D$ is a diagonal matrix with $a_{i i} \neq 0$ and $-L$ and $-U$ are strictly lower and upper triangular part of $A$. One of the iterative method is Jacobi (J) method expressed as:

$$
x^{(n+1)}=D^{-1}(L+U) x^{(n)}+D^{-1} b
$$

It has been proved that, if $A$ is strictly diagonally dominant (SDD) or irreducibly diagonally dominant, then the associated Jacobi iteration converges for any initial guess.

In this paper, we consider Refinement of Refinement of Jacobi iterative method, i.e. we mean that Second Refinement of Jacobi iterative method (SRJ). So here after, Refinement of Refinement of Jacobi iterative method (RRJ) we call Second Refinement of Jacobi iterative method (SRJ). Second Refinement of Jacobi Iterative method (SRJ method) is used to accelerate the convergence of basic Jacobi Iterative method.

The following definitions, lemma and theorem are important for our study used by [3], [4], [5], [2], [6].

Definition 1: A complex matrix $A \in \mathbb{C}^{n x n}$ is reducible if and only if there exists a permutation matrix $P$ (i.e. $P$ is obtained from the identity matrix $I$ by a permutation of the rows of $I$ ) and an integer $k \in\{1, \ldots, n-1\}$ such that

$$
P A P^{T}=\left(\begin{array}{cc}
A_{11} & A_{12} \\
O & A_{22}
\end{array}\right)
$$

Where $A_{11}$ is $k \times k$ and $A_{22}$ is $(n-k) \times(n-k)$. If $A$ is not reducible, then $A$ is said to be irreducible.

Definition 2: An $n \times n$ matrix $A=\left(a_{i j}\right)$ is said to be strictly diagonally dominant (SDD) if

$$
\left|a_{i i}\right|>\sum_{j=1, j \neq 1}^{n}\left|a_{i j}\right|
$$


Definition 3: If an $n \times n$ matrix $A=\left(a_{i j}\right)$ is said to be diagonally dominant (DD) if

$$
\left|a_{i i}\right| \geq \sum_{j=1, j \neq 1}^{n}\left|a_{i j}\right|
$$

Definition 4: $A$ is irreducibly diagonally dominant (IDD) if $A$ is irreducible and diagonally dominant, with strict inequality holding in Definition 2 for at least one $i$.

Definition 5: An $n \times n$ matrix $A=\left(a_{i j}\right)$ is said to be symmetric positive definite (SPD) if A is symmetric, $\left(A=A^{T}\right)$ and positive definite $x^{T} A x>0$ for all $x \neq 0$.

Definition 6: A matrix is said to be an M-matrix if it satisfies the following four properties:

1) $a_{i i}>0$ for $\mathrm{i}=1, \ldots, \mathrm{n}$

2) $a_{i j} \leq 0$ for $i \neq j, \mathrm{i}, \mathrm{j}=1, \ldots, \mathrm{n}$

3) $\mathrm{A}$ is nonsingular

4) $A^{-1} \geq O$

Alternatively, A matrix $A \in R^{n \times n}$ is said to be nonsingular Mmatrix if $A$ can be written as $A=s I-B$, where $B \geq O$ and $s>\rho(B)$.

Definition 7: The spectral radius of matrix $A$ is the largest absolute value of the eigenvalues of $A: \rho(A):=\max \{|\lambda|: \lambda \in$ $\sigma(A)\}$.

Definition 8: The rate of convergence of an iterative method is $R(G)=-\log _{10}[\rho(G)]$.

Lemma 1: The spectral radius satisfies the following rules

- $\rho(k A)=|k| \rho(A)$ for all $k \in \mathbb{C}$ and $A \in \mathbb{C}^{n \times n}$.

- $\rho\left(A^{k}\right)=(\rho(A))^{k}$ for all $k \in \mathbb{N}$ and $A \in \mathbb{C}^{n \times n}$.

- $\rho(A)=\rho\left(A^{H}\right)=\rho\left(A^{T}\right)$ for all $A \in \mathbb{C}^{n \times n}$.

Theorem 1: A linear iteration $\Phi(x, b)=M x+N b$ with the iteration matrix $M=M[A]$ is convergent if and only if $\rho(M)<$ 1 .

Theorem 2: Let $A=M-N$ be a regular splitting of the matrix $A$. Then $\rho\left(M^{-1} N\right)<1$ if and only if $A$ is nonsingular and $A^{-1} \geq O$.

Theorem 3: Let $A=\left(a_{i j}\right), B=\left(b_{i j}\right)$ be two matrices such that $A \leq B$ and $b_{i j} \leq O$ for all $i \neq j$. Then if $A$ is an M-matrix, so is the matrix $B$.

\section{ReFinement of JACOBI (RJ) Method}

The system of linear equations (1) is solved by different iterative methods. One of them is refinement of Jacobi (RJ) iterative method. This method was first proposed by Farhad Naeimi Dafchahi (2008). Let us show some steps to prove RJ method. Using equation (1), we have $(D-L-U) x=b$, where $A=D-L-U$. We obtain $D x=(L+U) x+b$ after taking $(L+$ $U) x$ to right side. Then we conclude $D x=(D-A) x+b$, where $L+U=D-A$. Finally, we have $D x=D x+b-A x$. Then we obtain $x=x+D^{-1}(b-A x)$ after rearranging and simplifying. Then we have $x^{(n+1)}=\tilde{x}^{(n+1)}+D^{-1}\left(b-A \tilde{x}^{(n+1)}\right)$. Next, we obtain $x^{(n+1)}=D^{-1}(L+U) x^{(n)}+D^{-1} b+D^{-1}\left[b-A\left(D^{-1}(L+\right.\right.$ $\left.\left.U) x^{(n)}+D^{-1} b\right)\right]$, where $\tilde{x}^{(n+1)}=D^{-1}(L+U) x^{(n)}+D^{-1} b$. Then we obtain $x^{(n+1)}=D^{-1}(L+U) x^{(n)}+D^{-1} b+D^{-1}[b-(D-L-$ $\left.U)\left(D^{-1}(L+U) x^{(n)}+D^{-1} b\right)\right]$. After simplification, we get:

$$
x^{(n+1)}=\left[D^{-1}(L+U)\right]^{2} x^{(n)}+\left[I+D^{-1}(L+U)\right] D^{-1} b
$$

Equation (3) is called Refinement of Jacobi (RJ).

\section{SECOND-REFINEMENT OF JACOBI METHOD (SRJ)}

This is our new modification of refinement of Jacobi iterative method. By taking: $x^{(n+1)}=\tilde{x}^{(n+1)}+D^{-1}\left(b-A \tilde{x}^{(n+1)}\right)$ substitute equation (3) on $\tilde{x}^{(n+1)}$. we get $x^{(n+1)}=\left[D^{-1}(L+\right.$ $U)]^{2} x^{(n)}+\left[I+D^{-1}(L+U)\right] D^{-1} b+D^{-1}\left(b-A\left\{\left[D^{-1}(L+\right.\right.\right.$ $\left.\left.U)]^{2} x^{(n)}+\left[I+D^{-1}(L+U)\right] D^{-1} b\right\}\right)$. After simplifying the above equation, we get:

$$
\begin{aligned}
x^{(n+1)}=\left[D^{-1}(L+U)\right]^{3} x^{(n)}+ \\
\\
\quad\left[I+D^{-1}(L+U)+\left(D^{-1}(L+U)\right)^{2}\right] D^{-1} b
\end{aligned}
$$

Equation (4) is called second-refinement of Jacobi (SRJ) iterative method.

\section{Convergence of SECond-Refinement of JACOBI METHOD (SRJ)}

Theorem 4: If A is a strictly diagonally dominant or an irreducibly diagonally dominant matrix, then the associated Jacobi iterations converge for any $x^{(0)}$.

Proof: See the proof in [2].

Theorem 5: If $A$ and $2 D-A$ are symmetric and positive definite matrices, then the Jacobi method is convergent for any initial guess.

Proof: Let $A$ and $2 D-A$ be SPD. We know $x^{*} A x>0$ and $x^{*}(2 D-A) x>0$, where $A=D-L-L^{T}$. $\Rightarrow D^{-1}\left(L+L^{T}\right) x=\lambda x \Rightarrow\left(L+L^{T}\right) x=\lambda D x \Rightarrow x^{*}\left(L+L^{T}\right) x=$ $\lambda x^{*} D x \Rightarrow x^{*} D x-x^{*} A x=\lambda x^{*} D x \Rightarrow x^{*} A x=(1-\lambda) x^{*} D x \Rightarrow$ $1-\lambda>0 \Rightarrow \lambda<1$

$\therefore \lambda<1$ $\circledast$

And we consider $x^{*}(2 D-A) x>0 \Rightarrow 2 x^{*} D x-x^{*} A x>0 \Rightarrow$ $x^{*} A x<2 x^{*} D x \Rightarrow(1-\lambda) x^{*} D x<2 x^{*} D x \Rightarrow 1-\lambda<2 \Rightarrow \lambda>-1$ $\therefore \lambda>-1 \ldots \ldots \ldots . . \circledast \circledast$

From $\circledast$ and $\circledast \circledast$, we get $-1<\lambda<1$. where $\lambda$ is the eigenvalues of $D^{-1}\left(L+L^{T}\right)$. Hence, $\rho\left(D^{-1}\left(L+L^{T}\right)\right)<1$.

Theorem 6: If $A$ is an M-matrix, then the Jacobi iterative method is convergent for any initial guess $x^{0}$.

Proof: Given $\mathrm{A}$ is M-matrix. Let $\mathrm{A}=\mathrm{M}-\mathrm{N}$. $\Rightarrow A=$ $D-L-U \Rightarrow M=D$ and $N=L+U \Rightarrow A \leq M \Rightarrow$ by Theorem $3 \mathrm{M}$ is M-matrix. $\Rightarrow M^{-1}>0$. On the other hand $N \geq 0$.

$\therefore A=M-N$ is a regular splitting of the matrix A. Having in mind that $A^{-1} \geq 0$ and Theorem 2 we deduce that $\rho\left(D^{-1}(L+\right.$ $U))<1$.

Theorem 7: If $A$ is row strictly diagonally dominant matrix, then the refinement of Jacobi (RJ) method converges for any arbitrary choice $x^{(0)}$

Proof: Assume $X$ is the real solution of the linear system (1). Because matrix $A$ is row strictly diagonally dominant, from Theorem 4 the Jacobi method is convergent then we can close $x^{(n+1)}$ to $X$ arbitrary thus we have $\left\|x^{(n+1)}-X\right\| \leq$ $\left\|x^{(n+1)}-X\right\|+\left\|D^{-1}\right\|\left\|b-A x^{(n+1)}\right\|$.

From $\left\|x^{(n+1)}-X\right\| \rightarrow 0$ we have $\left\|b-A x^{(n+1)}\right\| \rightarrow 0$ so $\left\|x^{(n+1)}-X\right\| \rightarrow 0 \Rightarrow \rho\left(D^{-1}(L+U)\right)<1$. So the refinement of Jacobi method converges to solution of linear system (1).

Theorem 8: If $A$ and $2 D-A$ are symmetric and positive definite matrices, then the refinement of Jacobi (RJ) iterative method converges for any initial guess $x^{(0)}$. 
Proof: Using Equation (2) and Theorem 5, we have $\rho\left(D^{-1}(L+U)\right)<1$. Since $A$ is SPD matrix. Let $X$ be the exact solution of (1). Then the Jacobi iterative method can be writen as $X=\left[I-D^{-1}(L+U)\right]^{-1} D^{-1} b$. Using equation (3) we have: $x^{(n+1)}=\left[D^{-1}(L+U)\right]^{2} x^{(n)}+\left[I+D^{-1}(L+U)\right] D^{-1} b$. Using this equation and exact solution $X$, we have:

$\Rightarrow X=\left[D^{-1}(L+U)\right]^{2} X+\left[I+D^{-1}(L+U)\right] D^{-1} b \Rightarrow X=[I-$ $\left.\left(D^{-1}(L+U)\right)^{2}\right]^{-1}\left[I+D^{-1}(L+U)\right] D^{-1} b$

After expanding and multiplying we get::

$X=I+D^{-1}(L+U)+\left[D^{-1}(L+U)\right]^{2}+\left[D^{-1}(L+U)\right]^{3}+\ldots$. $\Rightarrow X=\left[I-D^{-1}(L+U)\right]^{-1} D^{-1} b$

$\therefore X=\left[I-D^{-1}(L+U)\right]^{-1} D^{-1} b$ is consistent to (1) and Jacobi method. On the other hand

$x^{(n+1)}=\left[D^{-1}(L+U)\right]^{2} x^{(n)}+\left[I+D^{-1}(L+U)\right] D^{-1} b$

$=\left[D^{-1}(L+U)\right]^{4} x^{(n-1)}+\left[I+D^{-1}(L+U)+\left(D^{-1}(L+U)\right)^{2}+\right.$ $\left.\left(D^{-1}(L+U)^{3}\right)\right] D^{-1} b$

$=\left[D^{-1}(L+U)^{6}\right] x^{(n-2)}+\left[I+D^{-1}(L+U)+\left(D^{-1}(L+U)\right)^{2}+\right.$ $\left.\ldots+\left(D^{-1}(L+U)\right)^{5}\right] D^{-1} b$

$=$

$=\left[D^{-1}(L+U)\right]^{2(n+1)} x^{(0)}+\left[I+\ldots+\left(D^{-1}(L+U)\right)^{2 n+1}\right] D^{-1} b$

We have $\rho\left(D^{-1}(L+U)\right)<1$ since A is SPD.

$\Rightarrow \lim _{n \rightarrow \infty}\left[D^{-1}(L+U)\right]^{2(n+1)} x^{(0)}=0$.

$\Rightarrow \lim _{n \rightarrow \infty} x^{(n+1)}=\lim _{n \rightarrow \infty}\left[D^{-1}(L+U)\right]^{2(n+1)} x^{(0)}+\sum_{m=0}^{\infty}\left[D^{-1}(L+\right.$ $U)]^{m} D^{-1} b=0+\left[I-D^{-1}(L+U)\right]^{-1} D^{-1} b=\left[I-D^{-1}(L+\right.$

$U)]^{-1} D^{-1} b \rightarrow X$

$\Rightarrow \rho\left(\left(D^{-1}(L+U)\right)^{2}\right)=\left[\rho\left(D^{-1}(L+U)\right)\right]^{2}<1$.

Therefore, the refinement of Jacobi (RJ) iterative method is convergent.

Theorem 9: Let $A$ be an M-matrix. Then the refinement of Jacobi method is convergent for any initial guess $x^{(0)}$.

Proof: It follows from Theorem 6 and the proof of Theorem 7 above.

Theorem 10: If $A$ is strictly diagonally dominant matrix, then the second-refinement of Jacobi (SRJ) method converges for any choice of the initial approximation $x^{(0)}$.

Proof: Let $X$ be the real exact solution of linear system (1). We know that if $A$ is SDD matrix and RJ method is $\tilde{x}^{(n+1)}=\left[D^{-1}(L+U)\right]^{2} x^{(n)}+\left[I+D^{-1}(L+U)\right] D^{-1} b$, then using Theorem 4 and 7 the $\mathrm{J}$ and RJ methods are convergent. So $\tilde{x}^{(n+1)} \rightarrow X$. The SRJ method written as:

$x^{(n+1)}=\tilde{x}^{(n+1)}+D^{-1}\left(b-A \tilde{x}^{(n+1)}\right)$

$\Rightarrow x^{(n+1)}-X=\tilde{x}^{(n+1)}-X+D^{-1}\left(b-A \tilde{x}^{(n+1)}\right)$. Then

$\left\|x^{(n+1)}-X\right\|=\left\|\tilde{x}^{(n+1)}-X+D^{-1}\left(b-A \tilde{x}^{(n+1)}\right)\right\| \leq$

$\left\|\tilde{x}^{(n+1)-X}\right\|+\left\|D^{-1}\left(b-A \tilde{x}^{(n+1)}\right)\right\|$

$\Rightarrow\left\|x^{(n+1)}-X\right\| \leq\left\|\tilde{x}^{(n+1)}-X\right\|+\left\|D^{-1}\right\|\left\|b-A \tilde{x}^{(n+1)}\right\| \rightarrow$

$\|X-X\|+\left\|D^{-1}\right\|\|b-A X\|$

$=0+\left\|D^{-1}\right\|\|b-b\|=0+0=0$.

Then, $x^{(n+1)} \rightarrow X$

$\Rightarrow \rho\left(\left(D^{-1}(L+U)\right)^{3}\right)=\left(\rho\left(D^{-1}(L+U)\right)\right)^{3}<1$.

Therefore, the SRJ method is convergent.

Theorem 11: If $A$ and $2 D-A$ are SPD matrix, then the second-refinement of Jacobi iterative method is convergent for any initial guess $x^{(0)}$.
Proof: Using equation (2) and Theorem 5, we have $\rho\left(D^{-1}\left(L+L^{T}\right)\right)<1$. Let $\mathrm{X}$ be the exact solution of (1). Then the refinement of Jacobi iterative method can be written as $X=\left[I-D^{-1}\left(L+L^{T}\right)\right]^{-1} D^{-1} b$ if $x^{(n+1)} \rightarrow X$. Equation (4) is: $x^{(n+1)}=\left[D^{-1}(L+U)\right]^{3} x^{(n)}+\left(I+D^{-1}(L+U)+\left(D^{-1}(L+\right.\right.$ $\left.U))^{2}\right) D^{-1} b$. Now using equation (4) and the exact solution $X$, we have:

$\Rightarrow X=\left[D^{-1}(L+U)\right]^{3} X+\left(I+D^{-1}(L+U)+\left(D^{-1}(L+\right.\right.$ $\left.U))^{2}\right) D^{-1} b$

$\Rightarrow X=\left(I-\left(D^{-1}(L+U)\right)^{3}\right)^{-1}\left[I+D^{-1}(L+U)+\left(D^{-1}(L+\right.\right.$ $\left.U))^{2}\right] D^{-1} b$

$=\left[I+\left(D^{-1}(L+U)\right)^{3}+\left(D^{-1}(L+U)\right)^{6}+\ldots\right]\left[I+D^{-1}(L+U)+\right.$ $\left.\left(D^{-1}(L+U)\right)^{3}\right] D^{-1} b$

$=\left[I+D^{-1}(L+U)+\left(D^{-1}(L+U)\right)^{2}+\left(D^{-1}(L+U)\right)^{3}+\right.$ $\left.\left(D^{-1}(L+U)\right)^{4}+\ldots ..\right] D^{-1} b$

$=\left[I-D^{-1}(L+U)\right]^{-1} D^{-1} b$

$\therefore X=\left[I-D^{-1}(L+U)\right]^{-1} D^{-1} b$ is consistent to (1) and refinement of Jacobi method. On the other hand,

$x^{(n+1)}=\left[D^{-1}(L+U)\right]^{3} x^{(n)}+\left(I+D^{-1}(L+U)+\left(D^{-1}(L+\right.\right.$ $\left.U))^{2}\right) D^{-1} b$

$=\left[D^{-1}(L+U)\right]^{6} x^{(n-1)}+\left(I+D^{-1}(L+U)+\left(D^{-1}(L+U)\right)^{2}+\right.$ $\left.\left(D^{-1}(L+U)\right)^{3}+\left(D^{-1}(L+U)\right)^{4}+\left(D^{-1}(L+U)\right)^{5}\right) D^{-1} b$

$=\left[D^{-1}(L+U)\right]^{9} x^{(n-2)}+\left(I+D^{-1}(L+U)+\left(D^{-1}(L+U)\right)^{2}+\right.$ $\left.\left(D^{-1}(L+U)\right)^{3}+\ldots+\left(D^{-1}(L+U)\right)^{8}\right) D^{-1} b$

$=$

$=\left[D^{-1}(L+U)\right]^{3 n+3} x^{(0)}+\left(I+D^{-1}(L+U)+\left(D^{-1}(L+U)\right)^{2}+\right.$ $\left.\left(D^{-1}(L+U)\right)^{3}+\ldots+\left(D^{-1}(L+U)\right)^{3 n+2}\right) D^{-1} b$

We are given that $A$ is SPD then $\rho\left(D^{-1}\left(L+L^{T}\right)\right)<1$. Thus $\lim _{n \rightarrow \infty}\left[D^{-1}(L+U)\right]^{3 n+3}=0$.

$\Rightarrow \lim _{n \rightarrow \infty} x^{(n+1)}=\lim _{n \rightarrow \infty}\left[D^{-1}(L+U)\right]^{3 n+3}+\sum_{k=0}^{\infty}\left[D^{-1}(L+\right.$ $U)]^{n} D^{-1} b$

$=0+\left[I-D^{-1}(L+U)\right]^{-1} D^{-1} b=\left[I-D^{-1}(L+U)\right]^{-1} D^{-1} b \rightarrow$ $X$ $\Rightarrow \rho\left[\left(D^{-1}(L+U)\right)^{3}\right]=\left[\rho\left(D^{-1}(L+U)\right)\right]^{3}<1$.

Therefore, the second-refinement of Jacobi (SRJ) iterative method is convergent.

Theorem 12: Let $A=\left(a_{i j}\right)$ be an M-matrix. Then for a given natural number $m<n$, the second-refinement of Jacobi method converges for any choice of initial approximation $x^{(0)}$.

Proof: It follows from Theorem 6 and 9 and the proof of Theorem 10.

Theorem 13: The second-refinement of Jacobi method converges faster than the Jacobi and Refinement of Jacobi method when Jacobi method is convergent.

Proof: We can write equation (2) by $x^{(n+1)}=G x^{(n)}+C$, (3) by $x^{(n+1)}=G^{2} x^{(n)}+B$ and (4) by $x^{(n+1)}=G^{3} x^{(n)}+K$ where $G=D^{-1}(L+U), C=D^{-1} b, B=\left[I+D^{-1}(L+U)\right] D^{-1} b$ and $K=\left[I+D^{-1}(L+U)+\left(D^{-1}(L+U)\right)^{2}\right] D^{-1} b$. Given that, $\|G\|<1$. Let $X$ be the exact solution of (1).

$\Rightarrow X=G X+C, X=G^{2} X+B$ and $X=G^{3} X+K$ respectively.

Let us consider Jacobi method:

$\Rightarrow x^{(n+1)}=G x^{(n)}+C \Rightarrow x^{(n+1)}-X=G x^{(n)}-X+C$

$\Rightarrow x^{(n+1)}-X=G\left(x^{(n)}-X\right)+G X+C-X$

$\Rightarrow x^{(n+1)}-X=G\left(x^{(n)}-X\right)$ 
$\Rightarrow\left\|x^{(n+1)}-X\right\|=\left\|G\left(x^{(n)}-X\right)\right\| \leq\|G\|\left\|x^{(n)}-X\right\| \leq$ $\left\|G^{2}\right\|\left\|x^{(n-1)}-X\right\| \leq \ldots \leq\left\|G^{n}\right\|\left\|x^{(1)}-X\right\|$ $\Rightarrow\left\|x^{(n+1)}-X\right\| \leq\left\|G^{n}\right\|\left\|x^{(1)}-X\right\| \leq\|G\|^{n}\left\|x^{(1)}-X\right\|$

Now let us consider refinement of Jacobi method:

$\Rightarrow x^{(n+1)}=G^{2} x^{(n)}+B \Rightarrow x^{(n+1)}-X=G^{2} x^{(n)}-X+B$

$\Rightarrow x^{(n+1)}-X=G^{2}\left(x^{(n)}-X\right)+G^{2} X+B-X$

$\Rightarrow x^{(n+1)}-X=G^{2}\left(x^{(n)}-X\right)$

$\Rightarrow\left\|x^{(n+1)}-X\right\|=\left\|G^{2}\left(x^{(n)}-X\right)\right\| \leq\left\|G^{2}\right\|\left\|x^{(n)}-X\right\| \leq$ $\left\|G^{4}\right\|\left\|x^{(n-1)}-X\right\| \leq \ldots \leq\left\|G^{2 n}\right\|\left\|x^{(1)}-X\right\|$

$\Rightarrow\left\|x^{(n+1)}-X\right\| \leq\left\|G^{2 n}\right\|\left\|x^{(1)}-X\right\| \leq\|G\|^{2 n}\left\|x^{(1)}-X\right\|$

Again let us consider second-refinement of Jacobi method:

$\Rightarrow x^{(n+1)}=G^{3} x^{(n)}+K \Rightarrow x^{(n+1)}-X=G^{3} x^{(n)}-X+K$

$\Rightarrow x^{(n+1)}-X=G^{3}\left(x^{(n)}-X\right)+G^{3} X+K-X$

$\Rightarrow x^{(n+1)}-X=G^{3}\left(x^{(n)}-X\right)$

$\Rightarrow\left\|x^{(n+1)}-X\right\|=\left\|G^{3}\left(x^{(n)}-X\right)\right\| \leq\|G\|\left\|x^{(n)}-X\right\| \leq$ $\left\|G^{6}\right\|\left\|x^{(n-1)}-X\right\| \leq \ldots \leq\left\|G^{3 n}\right\|\left\|x^{(1)}-X\right\|$

$\Rightarrow\left\|x^{(n+1)}-X\right\| \leq\left\|G^{3 n}\right\|\left\|x^{(1)}-X\right\| \leq\|G\|^{3 n}\left\|x^{(1)}-X\right\|$

According to the coefficients of the above inequalities, we have $\|G\|^{3 n}<\|G\|^{2 n}<\|G\|^{n}$ since $\|G\|<1$.

Therefore, the second-refinement of Jacobi method converges faster than the Jacobi method and refinement of Jacobi method.

Theorem 14: If Jacobi method is convergent, then SecondRefinement of Jacobi (SRJ) method is convergent.

Proof: Given:- Jacobi method is convergent.

Required:- The Second Refinement of Jacobi method is convergent.

We know that Jacobi method is convergent $\Leftrightarrow \rho\left(D^{-1}(L+\right.$ $U))<1$

We want to show that Second Refinement of Jacobi method is convergent

$\Rightarrow \rho\left[\left(D^{-1}(L+U)\right)^{3}\right]=\rho\left[\left(D^{-1}(L+U)\right)\right]^{3}<1$

$\therefore$ The SRJ method is convergent.

\section{NumERICAL EXAMPLES}

Example 1: Consider the system of linear equations

$$
\left\{\begin{array}{l}
6 x_{1}+2 x_{2}+2 x_{3}=5 \\
2 x_{1}+8 x_{2}+2 x_{3}=6 \\
2 x_{1}+2 x_{2}+10 x_{3}=7
\end{array}\right.
$$

the coefficient matrix is both SDD and SPD matrix. To solve the system with tolerance 0.0001 by using Jacobi, refinement of Jacobi and second-refinement of Jacobi, would be shown in Table I.

TABLE I

SPECTRAL RADIUS

\begin{tabular}{|c|c|c|c|}
\hline Method & Jacobi & RJ & SRJ \\
\hline Spectral radius & 0.5146 & 0.2649 & 0.1362 \\
\hline Rate of convergence & 0.2885 & 0.5769 & 0.8658 \\
\hline
\end{tabular}

Table II shows that the second-refinement of Jacobi method (SRJ) is much better than Jacobi method $(\mathrm{J})$ and refinement of Jacobi (RJ) method. We think that it is almost half of faster than refinement of Jacobi method and one third of Jacobi method by Theorem 13 .

Example 2: Consider the system of linear equations

$$
\left\{\begin{array}{l}
6 x_{1}+4 x_{2}-x_{3}=9 \\
3 x_{1}+7 x_{2}+2 x_{3}=12 \\
-4 x_{1}+3 x_{2}+8 x_{3}=7
\end{array}\right.
$$

the coefficient matrix is SDD but not SPD matrix. To solve the system with tolerance 0.0001 by using Jacobi, refinement of Jacobi and second-refinement of Jacobi, would be shown in Table III.

Table IV shows us the new method (i.e. second refinement of Jacobi method) is much faster than Jacobi and refinement of Jacobi method.

Example 3: Consider the system of linear equations

$$
\left\{\begin{array}{l}
6 x_{1}+4 x_{2}+3 x_{3}=13 \\
4 x_{1}+5 x_{2}+2 x_{3}=11 \\
3 x_{1}+2 x_{2}+2 x_{3}=7
\end{array}\right.
$$

the coefficient matrix is both SPD but not SDD matrix. To solve the system with tolerance 0.0001 by using Jacobi, refinement of Jacobi and second-refinement of Jacobi, would be shown in Table V.

The solution of the above equation diverges from the exact solution. Therefore, no solution when we apply Jacobi method, refinement of Jacobi method and second-refinement of Jacobi method. Since the spectral radius of the iteration matrix is greater than one.

Example 4: Consider the system of linear equation

$\left\{\begin{array}{l}5 x_{1}+3 x_{2}+x_{3}=9 \\ 4 x_{1}-6 x_{2}+x_{3}=-1 \\ 2 x_{1}+x_{2}+4 x_{3}=7\end{array}\right.$

the coefficient matrix is SDD but not both PD and SPD matrix. To solve the system with tolerance 0.0001 by using Jacobi, refinement of Jacobi and second-refinement of Jacobi, would be shown in Table VI.

Table VII shows that the second-refinement of Jacobi iterative method is much better than Jacobi method and refinement of Jacobi method. We can also conclude that SRJ method minimizes iteration number to one-third as compared to $\mathrm{J}$ method. This is supported by Theorem 13 .

Example 5: Consider an M-matrix A (or 2-cyclic matrix A), which arises from discretization of the Poissons equations $\frac{\partial^{2} T}{\partial x^{2}}+\frac{\partial^{2} T}{\partial y^{2}}=f$, on the unit square as consider by Vatti [11], Datta [2] and Dafchahi [1]. Now consider $A X=b$, where $X=\left(\begin{array}{llllll}x_{1} & x_{2} & x_{3} & x_{4} & x_{5} & x_{6}\end{array}\right)^{T}$ and $b=\left(\begin{array}{llllll}1 & 0 & 0 & 0 & 0 & 0\end{array}\right)^{T}$ or

$$
\left(\begin{array}{cccccc}
4 & -1 & 0 & -1 & 0 & 0 \\
-1 & 4 & -1 & 0 & -1 & 0 \\
0 & -1 & 4 & 0 & 0 & -1 \\
-1 & 0 & 0 & 4 & -1 & 0 \\
0 & -1 & 0 & -1 & 4 & -1 \\
0 & 0 & -1 & 0 & -1 & 4
\end{array}\right)\left(\begin{array}{l}
x_{1} \\
x_{2} \\
x_{3} \\
x_{4} \\
x_{5} \\
x_{6}
\end{array}\right)=\left(\begin{array}{l}
1 \\
0 \\
0 \\
0 \\
0 \\
0
\end{array}\right)
$$

To solve the system with tolerance 0.0001 by using Jacobi, 
TABLE II

Numerical RESUlts of JACOBI, ReFinement of JACOBI AND SECOND REFINEMENT OF JACOBI IN EXAMPLE 1.

\begin{tabular}{|c|c|c|c|c|c|c|c|c|c|}
\hline \multicolumn{4}{|c|}{ First degree Jacobi (J) } & \multicolumn{2}{c|}{ Refinement of Jacobi (RJ) } & \multicolumn{3}{c|}{ SRJ } \\
\hline$n$ & $x_{1}{ }^{(n)}$ & $x_{2}{ }^{(n)}$ & $x_{3}{ }^{(n)}$ & $x_{1}{ }^{(n)}$ & $x_{2}{ }^{(n)}$ & $x_{3}{ }^{(n)}$ & $x_{1}{ }^{(n)}$ & $x_{2}{ }^{(n)}$ & $x_{3}{ }^{(n)}$ \\
\hline 0 & 0 & 0 & 0 & 0 & 0 & 0 & 0 & 0 & 0 \\
\hline 1 & 0.8333 & 0.7500 & 0.7000 & 0.3500 & 0.3667 & 0.3833 & 0.5833 & 0.5667 & 0.5567 \\
\hline 2 & 0.3500 & 0.3667 & 0.3833 & 0.4589 & 0.4650 & 0.4700 & 0.4890 & 0.4908 & 0.4921 \\
\hline 3 & 0.5833 & 0.5667 & 0.5567 & 0.4890 & 0.4908 & 0.4921 & 0.5025 & 0.5013 & 0.5011 \\
\hline 4 & 0.4589 & 0.4650 & 0.4700 & 0.4971 & 0.4976 & 0.4979 & 0.4998 & 0.4998 & 0.4999 \\
\hline 5 & 0.5217 & 0.5178 & 0.5152 & 0.4992 & 0.4994 & 0.4999 & 0.5000 & 0.5000 & 0.5000 \\
\hline 6 & 0.4890 & 0.4908 & 0.4921 & 0.4998 & 0.4998 & 0.4999 & & & \\
\hline 7 & 0.5057 & 0.5047 & 0.5040 & 0.4999 & 0.5000 & 0.5000 & & & \\
\hline 8 & 0.4971 & 0.4976 & 0.4979 & 0.5000 & 0.5000 & 0.5000 & & & \\
\hline 9 & 0.5015 & 0.5013 & 0.5011 & & & & & & \\
\hline 10 & 0.4992 & 0.4994 & 0.4994 & & & & & & \\
\hline 11 & 0.5004 & 0.5003 & 0.5003 & & & & & & \\
\hline 12 & 0.4998 & 0.4998 & 0.4999 & & & & & & \\
\hline 13 & 0.5001 & 0.5001 & 0.5001 & & & & & & \\
\hline 14 & 0.4999 & 0.5000 & 0.5000 & & & & & & \\
\hline 15 & 0.5000 & 0.5000 & 0.5000 & & & & & & \\
\hline
\end{tabular}

TABLE III

SPECTRAL RADIUS

\begin{tabular}{|c|c|c|c|}
\hline Method & Jacobi & RJ & SRJ \\
\hline Spectral radius & 0.7937 & 0.6299 & 0.5000 \\
\hline Rate of convergence & 0.1003 & 0.2007 & 0.3010 \\
\hline
\end{tabular}

[5] Y. Saad, Iterative methods for sparse linear systems. siam, 2003, vol. 82. [6] D. Young, Iterative solution of large linear systems. Elsevier, 2014.

refinement of Jacobi and second-refinement of Jacobi, would be shown in Table VIII.

Table 9a-c show that the new method SRJ gives the solutions at 7 th iteration where as Jacobi gives the solutions at 19th iteration and Refinement of Jacobi gives solutions at 10th iteration. So our new method minimizes the number of iterations by one-third of Jacobi method iterative method.

\section{CONClusions}

Obtaining solution of linear system of equations using numerical method is one of the most important subjects in Applied Science, Engineering and other Sciences. In this paper, we found that second-refinement of Jacobi iterative method for solving linear system of equations which minimizes the number of iteration almost by one-third of Jacobi and two-third of refinement of Jacobi iterative method. It also minimizes the spectral radius of $\mathbf{J}$ and $\mathrm{RJ}$ method. Its rate of convergence is larger than $\mathrm{J}$ and $\mathrm{RJ}$ method which means that our new method is fastest to convergence. A number of theorems have been considered to show the convergence of SRJ method and for verification, we also compare our results by taking a similar example considered by other researchers as shown in example 5.5.

\section{REFERENCES}

[1] A. Laskar and S. Behera, "Refinement of iterative methods for the solution of system of linear equations ax=b," IOSR Journal of Mathematics (IOSR$J M$ ), vol. 10, no. 3, pp. 70-73, 2014.

[2] R. Varga, Matrix iterative analysis. Springer Science \& Business Media, 2009, vol. 27.

[3] B. Datta, Numerical linear algebra and applications. Siam, 2010, vol. 116.

[4] W. Hackbusch, Iterative solution of large sparse systems of equations. Springer, 1994, vol. 95. 
TABLE IV

NUMERICAL RESUlTS OF JACOBI, REFINEMENT OF JACOBI AND SECOND REFINEMENT OF JACOBi IN EXAMPLE 2.

\begin{tabular}{|c|c|c|c|c|c|c|c|c|c|}
\hline \multicolumn{9}{|c|}{ First degree Jacobi (J) } & \multicolumn{3}{c|}{ Refinement of Jacobi (RJ) } & \multicolumn{3}{c|}{ SRJ } \\
\hline $\mathrm{n}$ & $x_{1}{ }^{(n)}$ & $x_{2}{ }^{(n)}$ & $x_{3}{ }^{(n)}$ & $x_{1}{ }^{(n)}$ & $x_{2}{ }^{(n)}$ & $x_{3}{ }^{(n)}$ & $x_{1}{ }^{(n)}$ & $x_{2}{ }^{(n)}$ & $x_{3}{ }^{(n)}$ \\
\hline 0 & 0 & 0 & 0 & 0 & 0 & 0 & 0 & 0 & 0 \\
\hline 1 & 1.5000 & 1.7143 & 0.8750 & 0.5030 & 0.8214 & 0.9821 & 1.1161 & 1.2181 & 0.8185 \\
\hline 2 & 0.5030 & 0.8214 & 0.9821 & 0.8243 & 1.0021 & 0.9762 & 0.9305 & 1.0276 & 0.9665 \\
\hline 3 & 1.1161 & 1.2181 & 0.8185 & 0.9305 & 1.0276 & 0.9665 & 0.9807 & 1.0221 & 0.9744 \\
\hline 4 & 0.8243 & 1.0021 & 0.9762 & 0.9663 & 1.0232 & 0.9732 & 0.9885 & 1.0102 & 0.9882 \\
\hline 5 & 0.9946 & 1.0821 & 0.9114 & 0.9810 & 1.0158 & 0.9818 & 0.9944 & 1.0052 & 0.9940 \\
\hline.. & $\ldots$ & $\ldots$ & $\ldots$ & $\ldots$ & $\ldots$ & $\ldots$ & $\ldots$ & $\ldots$ & $\ldots$ \\
\hline 12 & 0.9885 & 1.0102 & 0.9882 & 0.9993 & 1.0006 & 0.9993 & 1.0000 & 1.0000 & 1.0000 \\
\hline 13 & 0.9912 & 1.0083 & 0.9904 & 0.9996 & 1.0004 & 0.9995 & & & \\
\hline.. & $\ldots$ & $\ldots$ & $\ldots$ & $\ldots$ & $\ldots$ & $\ldots$ & & & \\
\hline 18 & 0.9972 & 1.0026 & 0.9970 & 1.0000 & 1.0000 & 1.0000 & & & \\
\hline.. & $\ldots$ & $\ldots$ & $\ldots$ & & & & & & \\
\hline 36 & 0.99996 & 1.00004 & 0.99995 & & & & & & \\
\hline 37 & 1.0000 & 1.0000 & 1.0000 & & & & & & \\
\hline
\end{tabular}

TABLE V

SPECTRAL RADIUS

\begin{tabular}{|c|c|c|c|}
\hline Method & Jacobi & RJ & SRJ \\
\hline Spectral radius & 1.4900 & 2.2202 & 3.3079 \\
\hline Rate of convergence & -0.173 & -0.346 & -0.52 \\
\hline
\end{tabular}

TABLE VI

SPECTRAL RADIUS

\begin{tabular}{|c|c|c|c|}
\hline Method & Jacobi & RJ & SRJ \\
\hline Spectral radius & 0.6227 & 0.3879 & 0.2415 \\
\hline Rate of convergence & 0.2057 & 0.4113 & 0.6171 \\
\hline
\end{tabular}

TABLE VII

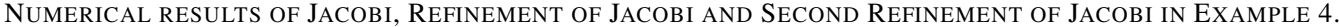

\begin{tabular}{|c|c|c|c|c|c|c|c|c|c|}
\hline \multicolumn{9}{|c|}{ First degree Jacobi(J) } & \multicolumn{2}{c|}{ Refinement of Jacobi(RJ) } & \multicolumn{3}{c|}{ SRJ } \\
\hline$n$ & $x_{1}{ }^{(n)}$ & $x_{2}{ }^{(n)}$ & $x_{3}{ }^{(n)}$ & $x_{1}{ }^{(n)}$ & $x_{2}{ }^{(n)}$ & $x_{3}{ }^{(n)}$ & $x_{1}{ }^{(n)}$ & $x_{2}{ }^{(n)}$ & $x_{3}{ }^{(n)}$ \\
\hline 0 & 0 & 0 & 0 & 0 & 0 & 0 & 0 & 0 & 0 \\
\hline 1 & 1.8000 & 0.1667 & 1.7500 & 1.3500 & 1.6583 & 0.8083 & 0.6433 & 1.2014 & 0.6604 \\
\hline 2 & 1.3500 & 1.6583 & 0.8083 & 0.9471 & 0.7056 & 1.1280 & 0.9884 & 1.1174 & 0.9280 \\
\hline 3 & 0.6433 & 1.2014 & 0.6604 & 0.9884 & 1.1174 & 0.9280 & 1.0182 & 1.0165 & 1.0020 \\
\hline 4 & 0.9471 & 0.7056 & 1.1280 & 1.0166 & 0.9587 & 1.0329 & 1.0050 & 0.9970 & 1.0047 \\
\hline 5 & 1.1510 & 0.9861 & 1.1001 & 0.9897 & 1.0125 & 0.9868 & 1.0002 & 0.9983 & 1.0010 \\
\hline 6 & 0.9884 & 1.1174 & 0.9280 & 1.0050 & 0.9971 & 1.0047 & 0.9997 & 0.9998 & 1.0000 \\
\hline 7 & 0.9440 & 0.9802 & 0.9765 & 0.9979 & 1.0002 & 0.9986 & 0.9999 & 1.0000 & 0.9999 \\
\hline 8 & 1.0166 & 0.9587 & 1.0329 & 1.0008 & 1.0003 & 1.0003 & 1.0000 & 1.0000 & 1.0000 \\
\hline.. & $\ldots$ & $\ldots$ & $\ldots$ & $\ldots$ & $\ldots$ & $\ldots$ & & & \\
\hline.. & $\ldots$ & $\ldots$ & $\ldots$ & $\ldots$ & $\ldots$ & $\ldots$ & & & \\
\hline 12 & 1.0050 & 0.9971 & 1.0047 & 1.0000 & 1.0000 & 1.0000 & & & \\
\hline.. & $\ldots$ & $\ldots$ & $\ldots$ & & & & & & \\
\hline.. & $\ldots$ & $\ldots$ & $\ldots$ & & & & & & \\
\hline 22 & 1.0000 & 0.9999 & 1.0000 & & & & & & \\
\hline 23 & 1.0000 & 1.0000 & 1.0000 & & & & & & \\
\hline
\end{tabular}

TABLE VIII

SPECTRAL RADIUS

\begin{tabular}{|c|c|c|c|}
\hline Method & Jacobi & RJ & SRJ \\
\hline Spectral radius & 0.6036 & 0.3643 & 0.2199 \\
\hline Rate of convergence & 0.2193 & 0.4385 & 0.6578 \\
\hline
\end{tabular}


TABLE IX

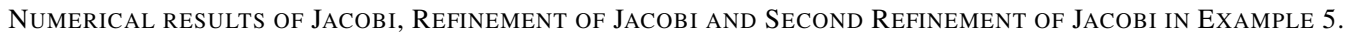

\begin{tabular}{|c|c|c|c|c|c|c|c|c|c|c|c|c|c|c|c|c|c|c|}
\hline \multicolumn{7}{|c|}{ Jacobi $(\mathrm{J})$ method } & \multicolumn{6}{|c|}{ Refinement of Jacobi (RJ) method } & \multicolumn{6}{|c|}{ Second-Refinement of Jacobi (SRJ) method } \\
\hline$n$ & $x_{1}{ }^{(n)}$ & $x_{2}{ }^{(n)}$ & $x_{3}{ }^{(n)}$ & $x_{4}{ }^{(n)}$ & $x_{5}{ }^{(n)}$ & $x_{6}{ }^{(n)}$ & $x_{1}{ }^{(n)}$ & $x_{2}{ }^{(n)}$ & $x_{3}{ }^{(n)}$ & $x_{4}{ }^{(n)}$ & $x_{5}{ }^{(n)}$ & $x_{6}{ }^{(n)}$ & $x_{1}{ }^{(n)}$ & $x_{2}{ }^{(n)}$ & $x_{3}{ }^{(n)}$ & $x_{4}{ }^{(n)}$ & $x_{5}{ }^{(n)}$ & $x_{6}{ }^{(n)}$ \\
\hline 0 & 0 & 0 & 0 & 0 & 0 & 0 & 0 & 0 & 0 & 0 & 0 & 0 & 0 & 0 & 0 & 0 & 0 & 0 \\
\hline 1 & 0.2500 & 0 & 0 & 0 & 0 & 0 & 0.25 & 0.0625 & 0 & 0.0625 & 0 & 0 & 0.2813 & 0.0625 & 0.0156 & 0.0625 & 0.0313 & 0 \\
\hline 2 & 0.25 & 0.0625 & 0 & 0.0625 & 0 & 0 & 0.2813 & 0.0820 & 0.0156 & 0.0781 & 0.0313 & 0.0117 & 0.2900 & 0.0891 & 0.0234 & 0.0833 & 0.0430 & 0.0166 \\
\hline 3 & 0.2813 & 0.0625 & 0.0156 & 0.0625 & 0.0313 & 0 & 0.2900 & 0.0891 & 0.0234 & 0.0833 & 0.0430 & \begin{tabular}{|l|}
0.0166 \\
\end{tabular} & 0.2942 & 0.0917 & 0.0275 & 0.0851 & 0.0488 & 0.0184 \\
\hline 4 & 0.2813 & 0.0820 & 0.0156 & 0.0781 & 0.0313 & 0.0117 & 0.2931 & \begin{tabular}{|l}
0.0917 \\
\end{tabular} & 0.0264 & 0.0851 & 0.0472 & 0.0184 & 0.2946 & 0.0930 & 0.0279 & 0.0860 & 0.0494 & 0.0193 \\
\hline 5 & 0.2900 & 0.0820 & 0.0234 & 0.0781 & 0.0430 & 0.0117 & 0.2942 & 0.0926 & 0.0275 & 0.0857 & 0.0488 & \begin{tabular}{|l}
0.0191 \\
\end{tabular} & 0.2948 & 0.0931 & 0.0281 & 0.0861 & 0.0496 & 0.0194 \\
\hline 6 & 0.2900 & 0.0891 & 0.0234 & 0.0833 & 0.0430 & 0.0166 & 0.2946 & 0.0930 & 0.0279 & 0.0860 & 0.0494 & \begin{tabular}{|l|}
0.0193 \\
\end{tabular} & 0.2948 & 0.0932 & 0.0281 & 0.0861 & 0.0497 & 0.0195 \\
\hline 7 & 0.2931 & 0.0891 & 0.0264 & 0.0833 & 0.0472 & 0.0166 & 0.2947 & 0.0931 & 0.0281 & 0.0861 & 0.0496 & 0.0194 & 0.2948 & 0.0 .0932 & 0.0282 & 0.0861 & 0.0497 & 0.0195 \\
\hline 8 & 0.2931 & 0.0917 & 0.0264 & 0.0851 & 0.0472 & 0.0184 & 0.2948 & 0.0931 & 0.0281 & 0.0861 & 0.0496 & \begin{tabular}{|l|l}
0.0194 \\
\end{tabular} & & & & & & \\
\hline 9 & 0.2942 & 0.0917 & 0.0275 & 0.0851 & 0.0488 & 0.0184 & 0.2948 & 0.0932 & 0.0281 & 0.0861 & 0.0497 & 0.0195 & & & & & & \\
\hline 10 & 0.2942 & 0.0926 & 0.0275 & 0.0857 & 0.0488 & 0.0191 & 0.2948 & 0.0932 & 0.0282 & 0.0861 & 0.0497 & 0.0195 & & & & & & \\
\hline.. & $\ldots$ & $\ldots$ & $\ldots$ & $\ldots$ & $\ldots$ & $\ldots$ & & & & & & & & & & & & \\
\hline &.. & $\ldots$ & $\ldots$ & $\ldots$ & $\ldots$ & - & & & & & & & & & & & & \\
\hline 18 & 0.2948 & 0.0932 & 0.0281 & 0.0861 & 0.0497 & 0.0195 & & & & & & & & & & & & \\
\hline 19 & 0.2948 & 0.0932 & 0.0282 & 0.0861 & 0.0497 & 0.0195 & & & & & & & & & & & & \\
\hline
\end{tabular}

